\section{The development of a measurement instrument focusing on team collaboration in patient transfer processes}

Patient transfer processes

\author{
Lilly-Mari Sten, Pernilla Ingelsson and Ingela Bäckström
} Department of Quality Management and Mechanical Engineering, Mid Sweden University, Östersund, Sweden, and

Marie Häggström

Department of Nursing Sciences, Mid Sweden University, Sundsvall, Sweden

\begin{abstract}
Purpose - Team collaboration is essential to ensure the quality of care and patient safety when critically ill patients are transferred from an intensive care unit (ICU) to a general ward. Measuring team collaboration in the patient transfer process can help gain insights into how team collaboration is perceived and how it can be improved. The purpose of this paper is to describe the development and testing of a questionnaire aiming to measure perceived team collaboration in the patient transfer process from ICU to the general ward. This study also aims to analyze the results to see how the survey could help improve team collaboration within ICU transitional care.

Design/methodology/approach - Statements, factors and main areas intended to measure perceived team collaboration were developed from a theory. The questionnaire was tested in two ICUs at two hospitals located in Sweden, and the results were analyzed statistically.

Findings - The results showed that the questionnaire could be used for measuring perceived team collaboration in this process. The results from the survey gave insights that can be useful when improving team collaboration in ICU transitional care.

Research limitations/implications - The collaboration between two research subjects, Nursing Science and Quality Management, has given new perspectives in how cultural and systemic differences and opportunities can help improving team collaboration in ICU transitional care, by shifting focus from the individual to team, culture, system, process and continuous improvement.

Practical implications - The developed questionnaire can be used to measure perceived team collaboration and to identify areas for improving team collaboration in the ICU transitional care process.

Originality/value - There is a sparse amount of research about measuring team collaboration in ICU transitional care, and this study contributes to filling this research gap.
\end{abstract}

Keywords Health care, Measurement, Quality improvement, Teamwork, Nursing management, QM

Paper type Research paper

(C) Lilly-Mari Sten, Pernilla Ingelsson, Ingela Bäckström and Marie Häggström. Published by Emerald Publishing Limited. This article is published under the Creative Commons Attribution (CC BY 4.0) licence. Anyone may reproduce, distribute, translate and create derivative works of this article (for both commercial and non-commercial purposes), subject to full attribution to the original publication and authors. The full terms of this licence may be seen at http://creativecommons.org/licences/by/4.0/ legalcode

The authors gratefully acknowledge the Family Kamprad Foundation for funding this research.

This paper forms part of a special section "QMOD-ICQSS Conference”, guest edited by Su Mi Dahlgaard-Park.
Received 9 April 2020 Revised 20 August 2020 Accepted 12 January 2021 
IJQSS

13,1

\section{Introduction}

Health-care organizations exist in a complex and constantly changing context in which patient needs frequently differ. Thus, to meet those differing requirements, health-care professionals play a crucial role in providing high quality and safe care. One way of giving better care is to bring health-care professionals together in interprofessional teams (Vyt, 2008). Coworkers collaborating in teams is a common way of working in the health-care sector and the quality of team collaboration has a direct impact on the quality of an organization's outcomes and performance (Boughzala and De Vreede, 2015). A large body of research indicates that effective team collaboration in health care is associated with increased patient safety (Manser, 2009; Häggström and Bäckström, 2014), reduced critical incidents and adverse advents (Manser, 2009), reduced patient mortality and sickness absence (Lyubovnikova et al., 2014), more effective use of resources and reduced health-care costs (Vyt, 2008).

Continuity of care is a term that has been used to describe the provision of care of an individual patient over time by bridging the care pathway (Crilly et al., 2006). An example of a gap in the care chain is when patients are transferred from a high-tech intensive care environment to an often less staffed ward environment, a process called intensive care unit (ICU) transitional care (Häggström et al., 2013). To ensure a safe and continuing care when critically ill patients are transferred from ICU to the general ward, coworkers need to collaborate both within and between hospital units. Gaps in the continuity of care because of a lack of team collaboration are a threat to patient safety.

Quality management $(\mathrm{QM})$ as a set of values, methods and tools can be very useful in the health-care sector for several reasons (Dahlgaard et al., 2011). First, QM has a system view and is a customer-focused management discipline that requires coworker involvement and teambuilding to succeed. The health-care sector has a long tradition of such a culture. Second, modern QM has a lot to offer health care in regard to values, methods and tools for working with continuous improvements (Dahlgaard and Dahlgaard-Park, 1999). Drotz and Pokinska (2014) deem that there seems to be a shift from a traditional focus on health-care professionals to a focus on process improvement and teamwork.

Measuring is an important way for organizations to benchmark their performance, both inside the organization and in comparison, with other organizations. Nursing Science, as a scientific subject, aims to make care better, safer and equal for both individual patients and groups of patients (Meleis, 2018). As team collaboration is a base for ICU transitional care and there is a sparse amount of research about measuring team collaboration within ICU transitional care, it is of great importance to gain knowledge about how to measure team collaboration and find ways to improve team collaboration and ICU transitional care. Manser (2009) deems that the challenge for future research is to further develop and validate instruments for team performance assessment in domains of health care.

The purpose of this paper is to describe the development and testing of a questionnaire aiming to measure perceived team collaboration in the patient transfer process from ICU to the general ward. This study also aims to analyze the results to see how the survey can help improve team collaboration within ICU transitional care. The purpose leads to the following research questions:

RQ1. How can an instrument that aims to measure perceived team collaboration in patient transfers from the ICU to the general ward be developed?

RQ2. How can a survey help improve team collaboration within ICU transitional care?

\section{Team and team collaboration in the health-care sector}

The phenomena team is described and defined in different ways. For example, can a team be described as individuals who share the responsibility and reward (or penalty) for the 
outcomes of the team's work (O'Leary et al., 2011). Hackman and Wageman (2005) define a team as a bounded set of individuals who work interdependently toward a shared outcome. According to Bronstein (2003), interdisciplinary processes among one or more professionals from different disciplines engaged in work-related activities should represent five core components: interdependence, newly created professional activities, flexibility, collective ownership of goals and reflection on the process.

West and Lyubovnikova (2012) have discovered that groups of professionals in the health-care sector perceive that they are working in a team but are not truly meeting the criteria for a "real team." A team that does not fulfil the criteria for a team can be defined as a "pseudo team" (Lyubovnikova et al., 2014). The "pseudo team" is a group of individuals who call themselves a team but who have different views of the objectives for the team and who are working alone or in separate formations to reach those dissimilar goals. "Pseudo teams" do not meet systematically to review team performance and adapt their goals and working processes accordingly. In a "pseudo team," the members do not know who the members of the team are. Creating this kind of distinction between a "real team" and a "pseudo team" gives the ability to understand why a team may not always succeed. Future research needs to better define the fundamental characteristics of teams in studies, to ensure that findings based on real teams, rather than pseudo teams, are accumulated (West and Lyubovnikova, 2013).

Hollenbeck et al. (2012) describe a typology for teams that place teams in three underlying dimensions. First, teams differ in skill differentiation. Previously, health-care teams used to be unidisciplinary with similar functional knowledge and conducted similar clinical tasks. Today, health-care teams are increasingly interdisciplinary. Interdisciplinary teams include members from different functional and clinical backgrounds working interdependently toward shared goals. The second dimension proposed by Hollenbeck is authority differentiation, which can be described as the degree to which decision-making is a responsibility for individual members, groups of the team or the collective as a whole. In interdisciplinary health-care teams, members are often involved in decision-making, which points to a lower authority differentiation. Third, some health-care teams maintain a relatively stable team structure over time, for example, team members working together over several years. Other teams may meet on occasion, for example, for an unusual surgical procedure. Teams with higher levels of temporal stability have the opportunity to develop effective team processes as team members develop a feeling of being a family. Thus, when researching and managing health-care teams, it is important to pay attention to the structural dimensions of skill differentiation, authority differentiation and temporal stability and how they impact team functioning and performance.

To achieve effective team collaboration in a dynamic health-care setting such as intensive care, where conditions frequently change and where there are different professional cultures, it is important to consider the specific requirements a team is confronted with. Sundstrom et al. (1990) call those kinds of coworker teams "action teams." Teams working as "action teams" in this kind of context may also vary depending on the situation. Effective team collaboration, both within and across teams, is a key feature for the delivery of high-quality patient care and patient safety (WHO, 2020). According to Häggström and Bäckström (2014), collaboration is a foundation for ICU transitional care and intertwines with the health-care chain. By working in teams, it is possible to better use specialist knowledge. In this study, team collaboration is defined as coworkers collaborating within and between hospital units based on shared views of person-centered care, 
IJQSS

13,1

Figure 1.

Important areas for team collaboration within ICU transitional care seen through the lens of $\mathrm{QM}$ continuous learning and sharing of knowledge, and with a focus on communication, coordination, structure and fact-based decisions.

\section{Measuring team collaboration within intensive care unit transitional care}

Bronstein (2003) developed a two-part model measuring interdisciplinary collaboration. The model was intended to be used in a context wherein social workers collaborated with other professionals to examine whether interdisciplinary collaboration was being carried out. An instrument named the Index for Interdisciplinary Collaboration (IIC) was developed from the model to measure the extent of collaboration between social workers and other professionals (Bronstein, 2002). The IIC was further modified by Oliver et al. (2007), who created a tool that could measure perceptions of collaboration by all members of a hospice team. This measuring tool was called the Modified Index for Interdisciplinary Collaboration. In a study by Kaissi et al. (2003), the attitudes and teamwork related to patient safety concerns in highrisk areas (i.e. ICUs) were measured. The conclusions of their study were that understanding team members' attitudes is the first necessary step in team training and that using the developed instrument could be an easy approach to achieve that goal.

\section{Seeing team collaboration within ICU transitional care through the lens of QM core values}

Presented below are important areas for team collaboration within ICU transitional care seen through the lens of QM. Those areas, found in previous research, show the presence of QM core values within health care and ICU transitional care (Figure 1).

\section{Person-centered care}

The QM core value "Focus on customers" and the concept of "Person-centered care" are similar in their approach. In QM, quality is defined by the customers and their needs and expectations. For organizations, this means to actively determine what customers want and how this can be fulfilled (Bergman and Klefsjö, 2010). In health care, person-centered care means putting the patient's needs and care as the overall priorities (Sharp et al., 2018). Person-centered care is a key indicator of quality care and a care strategy in many hospitals (ibid.).

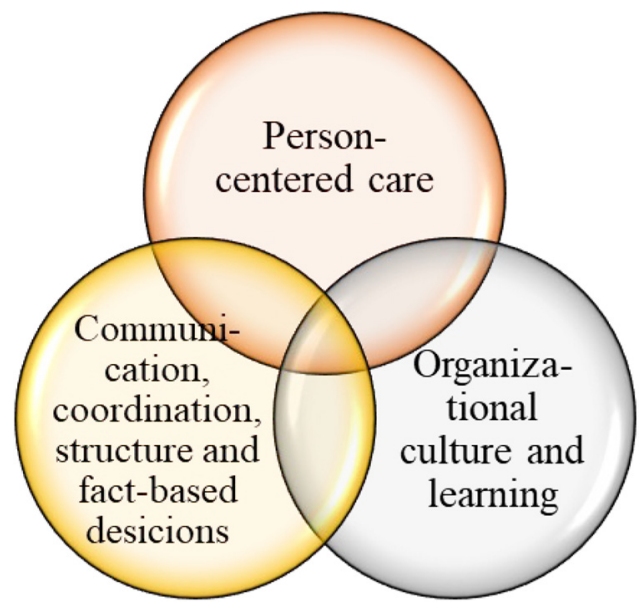


Performing person-centered care in an ICU may be challenging because of its complex context (Häggström et al., 2012). However, person-centered care has the potential to improve the outcomes of care. Person-centered care and individual care planning are seen as important parts of facilitating an ICU-patient's transition (ibid.). Teams collaborating within and between hospital units is an important part of achieving person-centered care. In this study, person-centered care is seen as collaboration in teams within and between hospital units based on the patient's individual needs and on how to create value for the patient.

\section{Organizational culture and continuous learning}

The culture of an organization can be seen as a system of shared values held by the individuals in the organization (Chatman and Eunyoung Cha, 2003). According to Henri (2006), a focus on organizational culture is important, as culture is a general factor that affects almost every part of organizational interactions. Schein (2009) argues that organizational culture exists on three levels: artifacts, espoused values and underlying assumptions.

There seems to be a difference between the care cultures of ICUs and general wards, which is something that affects both the staff's cooperation and the perception of the transfer for patients and relatives (Häggström et al., 2009; Häggström and Bäckström, 2014). ICU transitional care appears to be influenced by a good learning environment, procedures and policies regarding patient transfers and by teams collaborating across hospital boundaries (Singer et al., 2015). To develop a culture of collaboration and coordination in health care, there is a need for commitment to engage in shared learning and dialogue (Kydona et al., 2010). In this study, continuous learning within and between units is based on an organizational culture where team members learn from each other and understand strengths and weaknesses of the teams' members.

\section{Communication, coordination, structure and fact-based decisions}

It is important that patients' transfers from the ICU to the general ward are early and correctly prepared and occur at the right time, i.e. when there is no longer a need for intensive care (Whittaker and Ball, 2000; Chaboyer et al., 2005). Discharging patients from an ICU to a general ward is a high-risk event because of the complexity and acuity of the patients' medical conditions, the number and complexity of providers and the current care capacity on the general ward (Stelfox et al., 2015; van Sluisveld et al., 2017). The organizing and performing of patient transfers may be provided by ICU nurses, acute care nurses, physicians and other health-care professionals (Chaboyer et al., 2005). A lack of team collaboration or poor quality of coordination can generate mistakes and unnecessary costs in health care. Readmissions to the ICU are associated with increased mortality related to the patient's already vulnerable condition (Renton et al., 2011) and a risk that other patients at the ward are being unintentionally neglected by the ward nurses (Kauppi et al., 2018). Deficiencies in the organization, such as inadequate procedures and planning and lack of information, communication or collaboration, are also reasons for threats to patient safety (Häggström et al., 2009; Häggström et al., 2012; Lin et al., 2013). According to Häggström and Bäckström (2014), a successful ICU transitional process involves coordination, optimal timing, early mobilization, participation and a multidiscipline approach. In a literature review by Reader et al. (2009), four main processes for teamwork were identified, i.e. communication, coordination, leadership and decision-making. A prototype framework explaining team performance in the ICU was developed using these categories.

Structure, discharge guidelines and policies are important in the management of patient transfers (Häggström et al., 2009; Häggström et al., 2013). The discharge process should be 
IJQSS

13,1

supported by tools such as standard protocols and structure to ensure patient safety during the transfer and when to make decisions about the discharge (Lin et al., 2013). In QM, one of the core values is "Base decisions on facts" (Bergman and Klefsjö, 2010). This means that both numerical and verbal data are needed as well as systematic tools for the structure of these data (ibid.). Random factors should not determine which decisions that are made. From previous research, communication, coordination, structure and fact-based decisions seem to be important aspects when teams are collaborating within ICU transitional care.

\section{Methods}

Research project

The research presented is conducted as part of a research project called "Increased Quality and Efficiency in Patient Transfers." This project is financed by The Kamprad Family Foundation and was initiated in January 2018. The overall purpose of the project is to gain new knowledge about how efficiency and quality in patient transfers within ICU transitional care can be improved, focusing on leadership, continuity of care, safety culture and learning. The purpose will be achieved through cooperation between the two research subjects, namely, QM and Nursing Science.

\section{Participants of the survey}

The participants of the survey worked at two middle-sized hospitals located in rural areas in the middle of Sweden. Their professions were nurses, physicians and assistant nurses at ICUs at the two hospitals. Leaders were not included but will be a part of analyzing participants from the whole process in a later study.

In total, 152 ICU coworkers received the questionnaire, and 84 of the ICU coworkers answered. The response rate was $55.3 \%$ for the randomly selected ICU coworkers. The questionnaire was handed out and answered at staff meetings for nurses, assistant nurses and physicians at the two ICUs. All of the coworkers that joined the staff meetings answered the questionnaire, which was approximately $35 \%$ of total coworkers. The staff who did not participate at the meetings received the questionnaire from their leaders $(65 \%)$.

\section{Ethical considerations}

The project has been ethically evaluated by the Swedish Ethical Review Authority (Ref. 2018-159-31M). Coworkers answering the questionnaire were informed about participating in the study in accordance with verbal and written criteria. They were informed about confidentiality and their rights to withdraw their participation without giving any reason.

\section{Development of a questionnaire}

A questionnaire was developed in three stages as follows:

(1) Initially, a literature search was undertaken to determine the current opinion on team collaboration within ICU transitional care and how QM core values could be seen in this particular literature.

(2) From the theory about ICU transitional care, 20 statements were developed. Ten statements were about team collaboration within a hospital unit, and ten statements were about team collaboration between hospital units.

(3) Each group of ten statements (referred to as main areas) was divided into three factors, with three to four statements in each factor. The main area is a group of factors connecting to each other. The main areas were "Team collaboration within a hospital unit" and "Team collaboration between hospital units." The factors for 
the two main areas were named "Person-centered care," "Continuous learning" and "Prerequisites for successful patient transfers" (Tables 1 and 2). A factor is a group of statements connecting to each other.

The developed main areas, factors and statements about team collaboration within and between hospital units were included in an existing questionnaire assessing quality culture. The earlier questionnaire was used and tested in different settings and contexts (see, for instance, Bäckström and Ingelsson, 2016). This original questionnaire consisted of 36 statements representing 12 different factors. In this earlier questionnaire, a factor measuring "Customer focus" was used; however, in this study, this factor was redesigned to fit healthcare settings and renamed "Person-centered care." The new extended measuring instrument was called "Assessing Quality Culture Health Care edition”. The questionnaire included team collaboration within and between units and consisted of 50 statements and 16 different factors. However, this paper only contains the analysis of the factors concerning team collaboration within and between hospital units and can be understood independently of the total questionnaire. The analysis of the total questionnaire will be presented in another paper.

The questionnaire had a seven-point Likert agreement scale ranging from "Agree strongly" to "Disagree strongly." There was also an opportunity for the respondent to answer, "Do not know/do not want to answer." The questionnaire started with questions about the coworker's hospital unit, profession, number of years at the specific unit and education. There was no question about gender, as the number of men answering the questionnaire was few; thus, including gender could risk revealing anonymity.

\section{Data collection and data analysis}

Data were collected at two ICUs at the two hospitals during January and March 2019.

The results from measuring coworker's perceived team collaboration in ICU transitional care at the studied hospitals will be presented below in the result section. The results from the questionnaire were analyzed using statistical package for social sciences (SPSS), and the analysis was carried out in the following four stages.

(1) Cronbach's alpha, mean and standard deviation:

- First, the internal consistency reliability was calculated for the six new factors about team collaboration by calculating the Cronbach's alpha coefficient for each of the factors. Internal consistency describes the extent to which all the items in a test measure the same concept and is therefore connected to the items' interrelatedness within a test (Tavakol and Dennick, 2011). As the factors consisted of three to four statements, a value of 0.6 or more was seen as acceptable (Hair, 2019), as the coefficient tends to increase with the number of items or statements.

\begin{tabular}{ll}
\multicolumn{2}{c}{ Main areas for developed factors about team collaboration } \\
Team collaboration within a hospital unit & Team collaboration between hospital units \\
\hline $\begin{array}{l}\text { Person-centered care within a hospital unit } \\
\text { Continuous learning within a hospital unit }\end{array}$ & $\begin{array}{l}\text { Person-centered care between hospital units } \\
\text { Continuous learning between hospital units }\end{array}$ \\
$\begin{array}{l}\text { Prerequisites for successful patient transfers within a } \\
\text { hospital unit }\end{array}$ & $\begin{array}{l}\text { Prerequisites for successful patient transfers } \\
\text { between hospital units }\end{array}$ \\
\hline
\end{tabular}

Table 1.

Developed main areas and factors about team collaboration 
IJQSS
13,1

52

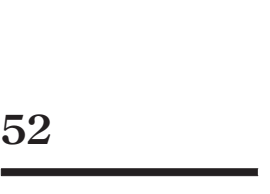

Factors

Person-centered care within a hospital unit

Statements Our mission at the unit is based on the patient's individual needs

What we do in the care team is based on the patients' individual needs

At our department, care teams know what creates value for each individual patient
Team collaboration within a hospital unit

Prerequisites for successful patient transfers within a hospital unit hospital unit

At our unit, the care teams see At our unit, we its part in the care chain and act on it communicate with each other within the care teams how to best transfer patients from ICU to general ward

In our unit, the care teams take advantage of combined competences and learn from each other

At our unit, the care teams have a good understanding of their own strengths and weaknesses
At our unit, care teams coordinate its activities in the best possible way, to transfer patients from ICU to general ward In our unit, decisions about patients' transfers are well grounded and based on facts At our unit, the care teams follow a clear structure to best transfer patients from ICU to general ward
Main area

Factors

Statements

At our hospital we have a common goal based on patient's individual needs

When we collaborate in care teams across departmental boundaries, we start from the patient's individual needs

When we collaborate in care teams across departmental boundaries, we know how we together create value for each individual patient
Team collaboration between hospital units

Continuous learning between
hospital units
In the care chain, care teams at
different units collaborate to
take advantage of the overall
competence

In the care chain, the care teams at different units learn from each other

In the care chain, the care teams at different units have a good understanding of each other's strengths and weaknesses
Prerequisites for successful patient transfers between hospital units

In the care chain, care teams from different units communicate with each other to best transfer patients from ICU to general ward In the care chain, care teams from different units coordinate their activities to best transfer patients from ICU to general ward In the care chain, the decisions about patients' transfers are well grounded and based on facts

In the care chain, the care teams follow a clear structure to best transfer patients from ICU to general ward 
- Preliminary analyses were performed to ensure no violation of normality, linearity and homoscedastic by scatterplots.

- The mean and standard deviation were calculated for each of the six factors by hospital ICU and by profession.

(2) Significant differences between ICUs, professions and factors:

- To analyze if there were significant differences between the two ICUs at the two hospitals, an independent-samples $t$-test was conducted with a significance level of $<0.05$.

- A one-way between-groups ANOVA was carried out to identify if the means between professions (physicians, nurses and assistant nurses) differed significantly (significant level 0.05).

- A paired sample $t$-test was conducted to investigate whether there was a significant difference between the perspectives within and between units with regard to the factors "Person-centered care," "Continuous learning" and "Prerequisites for successful patient transfers."

(3) Correlations between main areas and factors:

- Correlations between the factors were studied using Pearson's Product-Moment Correlation and Spearman's Coefficient of Rank Correlation. Correlations with an $R$-value above 0.50 (significant level 0.01 ) were considered high.

(4) Missing value analysis:

- Finally, missing values were analyzed for the factors to determine whether there were differences between the factors in answering the questionnaire.

\section{Results}

The results are presented in accordance with the process mentioned in the Methods section (Table 3).

\section{Cronbach's alpha, mean and standard deviation}

The SPSS calculation of Cronbach's alpha for the six factors about team collaboration produced values from 0.60 to 0.86 . As all of the factors reached a value of 0.6 , no further analysis was conducted at the statement level.

The mean values for the factors ranged from 4.14 to 5.77. The factor "Person-centered care within unit" had the highest mean, and the factor "Continuous learning between units" had the lowest mean.

\begin{tabular}{|c|c|c|c|c|c|}
\hline Factors & $\begin{array}{c}\text { No. of } \\
\text { statements }\end{array}$ & $\begin{array}{c}\text { Cronbach's } \\
\text { alpha }\end{array}$ & Mean & SD & Table 3. \\
\hline Person-centered care within a hospital unit & 3 & 0.78 & 5.77 & 0.79 & mean value and \\
\hline Continuous learning within a hospital unit & 3 & 0.60 & 5.34 & 0.87 & standard deviation \\
\hline $\begin{array}{l}\text { Prerequisites for successful patient transfers within } \\
\text { a hospital unit }\end{array}$ & 4 & 0.86 & 5.23 & 1.02 & for the developed \\
\hline Person-centered care between hospital units & 3 & 0.77 & 5.04 & 1.09 & factors regarding \\
\hline Continuous learning between hospital units & 3 & 0.74 & 4.14 & 1.13 & eam collaboration \\
\hline $\begin{array}{l}\text { Prerequisites for successful patient transfers between } \\
\text { hospital units }\end{array}$ & 4 & 0.85 & 4.54 & 1.08 & $\begin{aligned} \text { tween } \\
\text { units }\end{aligned}$ \\
\hline
\end{tabular}




\section{IJQSS \\ 13,1}

Table 4.

Mean values and standard deviations for the measured factors divided by ICU and hospital
The standard deviation values ranged from 0.79 to 1.13. The factor "Person-centered care within unit" had the lowest standard deviation, and the factor "Continuous learning between units" had the highest standard deviation. The factor "Person-centered care within unit" had higher mean and lower standard deviation than "Person-centered care between units." "Continuous learning within unit" had higher mean and lower standard deviation than "Continuous learning between units."

The means were higher and the standard deviations were lower for the factors concerning "Team collaboration within unit" compared to the factors concerning "Team collaboration between units" (Table 3 ).

The mean values and standard deviations were calculated for the two ICUs at the two hospitals to determine whether the results from the ICUs differed from each other. The mean values were generally higher at the ICU at Hospital 2. The values for standard deviation varied between the two hospitals' ICUs (Table 4).

The mean values and standard deviations were calculated by profession. The mean values for assistant nurses were generally higher than those for physicians and nurses. The values for the standard deviations varied between the professions (Table 5).

\section{Significant differences between intensive care units, professions and factors}

An independent-samples $t$-test was conducted to compare the factor scores for the two ICUs at the two hospitals. The factors with the highest mean difference between the two groups were the factors for "Continuous learning within unit" (0.347) and "Prerequisites for successful patient transfers within unit" $(0.449)$. There were no significant differences in scores between the two ICUs (ranging from 0.05 to 0.82 ), and therefore, the results were treated as the same group. Further analyses were performed with this assumption (Table 6).

The factor scores for the three different professions were tested with a one-way betweengroups ANOVA. Post hoc comparisons using the Tukey honest significant difference test

\begin{tabular}{lcccc}
\hline & \multicolumn{3}{c}{ ICU hospital 1 } & \multicolumn{2}{c}{ ICU hospital 2 } \\
Factors & Mean & SD & Mean & SD \\
\hline Person-centered care within a hospital unit & 5.68 & 0.75 & 5.84 & 0.83 \\
Continuous learning within a hospital unit & 5.15 & 0.78 & 5.50 & 0.93 \\
Prerequisites for successful patient transfers within a hospital unit & 4.99 & 1.17 & 5.44 & 0.84 \\
Person-centered care between hospital units & 5.12 & 1.08 & 4.97 & 1.11 \\
Continuous learning between hospital units & 4.11 & 1.37 & 4.17 & 0.90 \\
Prerequisites for successful patient transfers between hospital units & 4.46 & 1.20 & 4.60 & 0.98 \\
\hline
\end{tabular}

\section{Table 5.}

Mean values and standard deviations for the measured factors divided by profession

\begin{tabular}{|c|c|c|c|c|c|c|}
\hline \multirow[b]{2}{*}{ Factors } & \multicolumn{2}{|c|}{ Physicians } & \multicolumn{2}{|c|}{ Nurses } & \multicolumn{2}{|c|}{ Assistant nurse: } \\
\hline & Mean & $\mathrm{SD}$ & Mean & $\mathrm{SD}$ & Mean & $\mathrm{SD}$ \\
\hline Person-centered care within a hospital unit & 5.54 & 0.92 & 5.73 & 0.67 & 5.91 & 0.80 \\
\hline Continuous learning within a hospital unit & 5.27 & 0.93 & 5.38 & 0.84 & 5.33 & 0.90 \\
\hline $\begin{array}{l}\text { Prerequisites for successful patient transfers within a } \\
\text { hesnital unit }\end{array}$ & & & & & & \\
\hline hospital unit & 5.12 & 0.86 & 5.12 & 0.99 & 5.39 & 1.13 \\
\hline Person-centered care between hospital units & 4.92 & 1.19 & 4.60 & 1.00 & 5.52 & 0.95 \\
\hline Continuous learning between hospital units & 3.99 & 0.97 & 3.99 & 0.91 & 4.40 & 1.39 \\
\hline $\begin{array}{l}\text { Prerequisites for successful patient transfers between } \\
\text { hospital units }\end{array}$ & 4.42 & 0.94 & 4.21 & 0.85 & 4.91 & 1.25 \\
\hline
\end{tabular}


indicated that the mean scores between nurses and assistant nurses according to the factors for "Person-centered care between units" and "Prerequisites for successful patient transfers between units" differed significantly (Table 7).

A paired sample $t$-test was conducted to evaluate the difference in mean scores between the two main areas, namely, "Team collaboration within and between units," for the factors of "Person-centered care," "Continuous learning" and "Prerequisites for successful transfers." The results showed a statistically significant difference, with higher mean scores for the factors within units compared to between units (Table 8).

\section{Patient transfer processes}

\section{Correlations between main areas and factors}

Pearson's Product-Moment Correlation was used to determine whether and how the main areas and factors correlated with each other. Spearman's Coefficient of Rank Correlation was also calculated. Both measures generated similar results. Correlations with an $R$-value above 0.50 (significant level 0.01) were considered high (Tables 9 and 10).

\begin{tabular}{|c|c|c|c|c|c|c|}
\hline \multirow[b]{2}{*}{ Factor } & \multicolumn{2}{|c|}{$\begin{array}{l}\text { Levene's test for } \\
\text { equality of variances }\end{array}$} & \multicolumn{4}{|c|}{$\begin{array}{c}t \text {-test for } \\
\text { equality of means }\end{array}$} \\
\hline & & $\begin{array}{c}\text { Mean } \\
\text { difference }\end{array}$ & Sig. & $t$ & df & $\begin{array}{c}\text { Sig. } \\
\text { (two-tailed) }\end{array}$ \\
\hline Person-centered care within a hospital unit & 0.092 & 0.161 & 0.763 & -0.928 & 81 & 0.36 \\
\hline $\begin{array}{l}\text { Continuous learning within a hospital unit } \\
\text { Prerequisites for successful patient transfers }\end{array}$ & 0.154 & 0.347 & 0.696 & -1.838 & 82 & 0.07 \\
\hline within a hospital unit & 7.855 & 0.449 & 0.006 & -1.999 & 68 & 0.05 \\
\hline Person-centered care between hospital units & 0.001 & 0.146 & 0.979 & 0.596 & 79 & 0.55 \\
\hline Continuous learning between hospital units & 5.096 & 0.063 & 0.027 & -0.230 & 55 & 0.82 \\
\hline $\begin{array}{l}\text { Prerequisites for successful patient transfers } \\
\text { between hospital units }\end{array}$ & 2.220 & 0.137 & 0.140 & -0.557 & 76 & 0.58 \\
\hline
\end{tabular}

Note: Significant difference at $<0.05$

Table 6.

No statistically significant differences between factors for the two

ICUs at the two hospitals using an independent samples $t$-test

\begin{tabular}{llc}
\hline Factors & Professions (mean) & Sign. \\
\hline Person-centered care between hospital units & Nurses (4.60) & 0.002 \\
Prerequisites for successful patient transfers between hospital units & Assistants nurses (5.52) & Nurses (4.21) \\
& Assistants nurses (4.91) & 0.032
\end{tabular}

Table 7.

Statistically significant differences between professions using one-way betweengroups ANOVA

\begin{tabular}{|c|c|c|c|c|c|}
\hline Factor & $\begin{array}{c}\text { Mean } \\
\text { difference }\end{array}$ & $t$ & df & $\begin{array}{c}\text { Sig. } \\
\text { (two-tailed) }\end{array}$ & Table 8. \\
\hline $\begin{array}{l}\text { Person-centered care within a hospital unit } \\
\text { Person-centered care between hospital units }\end{array}$ & 0.740 & 7.444 & 79 & 0.000 & $\begin{array}{r}\text { Statistically } \\
\text { significant }\end{array}$ \\
\hline $\begin{array}{l}\text { Continuous learning within a hospital unit } \\
\text { Continuous learning between hospital units }\end{array}$ & 1.224 & 10.048 & 74 & 0.000 & $\begin{array}{l}\text { differences between } \\
\text { factors for the two }\end{array}$ \\
\hline $\begin{array}{l}\text { Prerequisites for successful patient transfers within a hospital unit } \\
\text { Prerequisites for successful patient transfers between hospital units }\end{array}$ & 0.705 & 8.248 & 77 & 0.000 & $\begin{array}{l}\text { main areas using a } \\
\text { paired sample } t \text {-test }\end{array}$ \\
\hline
\end{tabular}


IJQSS

13,1

56

Table 9.

Correlation between the main areas
The correlation between the main areas was 0.682 .

The highest correlation was found between the factors "Prerequisites for successful patient transfers within unit" and "Prerequisites for successful patient transfer between units" (0.736).

From the table above, correlation patterns were designed to visualize the correlations between the factors in the two main areas (Figure 2).

\section{Missing value analysis}

An analysis was conducted to determine the percentage of missing values for the six factors. Missing values can occur randomly or in a systematic pattern. Tables 11-13 only show factors with missing values and the percentage of missing values.

The factor with the highest missing values was "Continuous learning between units" at $10.7 \%$. There are missing values for all factors concerning the main area "Team collaboration between units" (Table 11).

Table 12 shows factors with missing values divided by profession. Missing values are highest for assistant nurses and for the factor "Continuous learning between units" (20.0\%). Physicians had the lowest missing values and for the factor "Person-centered care within unit."

Table 13 shows missing values for factors divided by ICU and hospital. ICU hospital 1 had the highest amount of missing values and for the factor "Continuous learning between units" $(12.8 \%)$ (Table 13$)$.

\begin{tabular}{lcc}
\hline Main areas & 1 & 2 \\
\hline 1. Team collaboration within a hospital unit & $\mathrm{X}$ & $0.682^{* * *}$ \\
2. Team collaboration between hospital units & & $\mathrm{X}$
\end{tabular}

Note: **Correlation is significant at the 0.01 level (two-tailed) using Pearson's Product-Moment Correlation
Figure 2.

Correlation patterns between the six measured factors and the two main areas

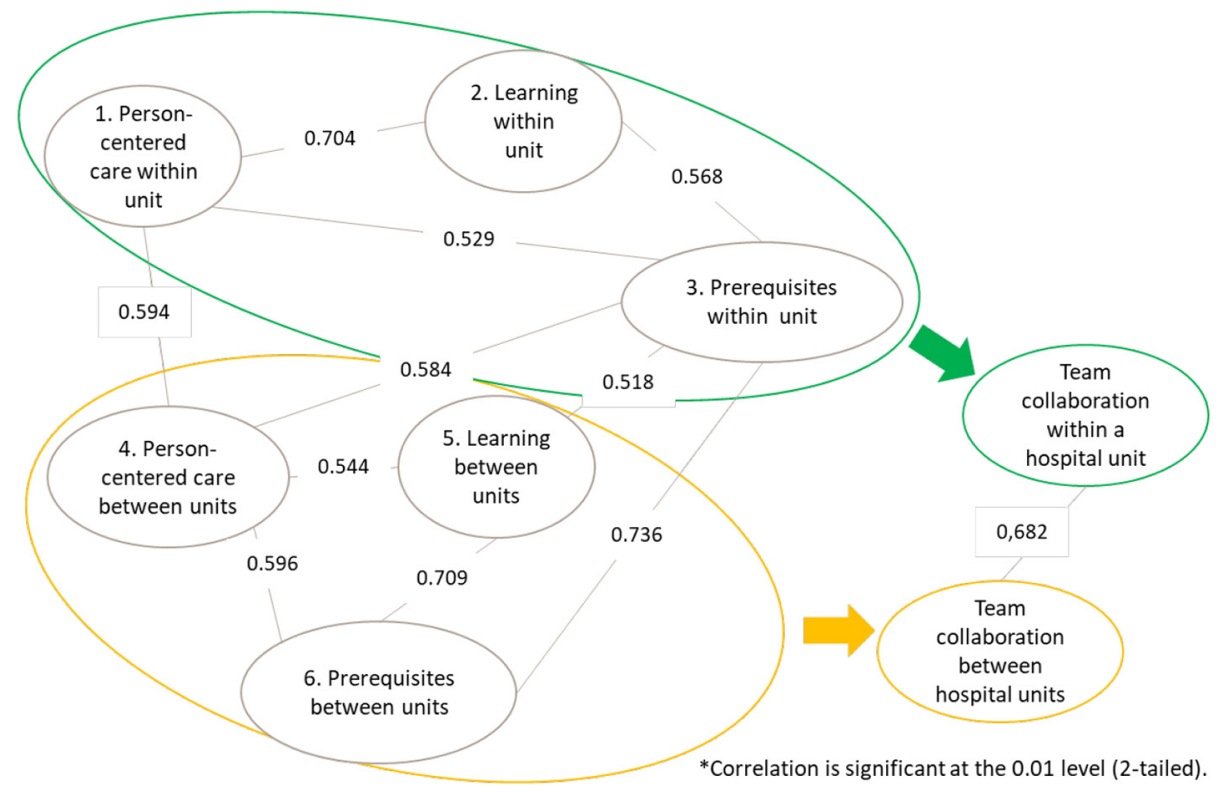




\section{Discussion and analysis}

The purpose of this paper was to describe the development and testing of a questionnaire aiming to measure perceived team collaboration in the patient transfer process ICU to the general ward. Six factors divided into two main areas measuring team collaboration within ICU transitional care were developed. Statements that formed the factors derived from literature about ICU transitional care and QM (i.e. Häggström et al., 2012; Häggström and Bäckström, 2014; Manser, 2009; Reader et al., 2009; Bergman and Klefsjö, 2010; van Sluisveld et al., 2017). Statistical analysis of the developed factors about team collaboration showed internal consistency and thus that the results could be analyzed at a factor level.

\begin{tabular}{|c|c|c|c|c|c|c|}
\hline Factors & 1 & 2 & 3 & 4 & 5 & 6 \\
\hline 1. Person-centered care within a hospital unit & $\mathrm{X}$ & $0.704^{* *}$ & $0.529 * *$ & $0.594 * *$ & - & - \\
\hline 2. Continuous learning within a hospital unit & & $\mathrm{X}$ & $0.568 * *$ & - & - & - \\
\hline $\begin{array}{l}\text { 3. Prerequisites for successful patient transfers within } \\
\text { a hospital unit }\end{array}$ & & & $\mathrm{X}$ & $0.584^{* *}$ & $0.518^{* *}$ & $0.736^{* *}$ \\
\hline 4. Person-centered care between hospital units & & & & $\mathrm{X}$ & $0.544 * *$ & $0.596 * *$ \\
\hline 5. Continuous learning between hospital units & & & & & $\mathrm{X}$ & $0.709 * *$ \\
\hline $\begin{array}{l}\text { 6. Prerequisites for successful patient transfers between } \\
\text { hospital units }\end{array}$ & & & & & & $\mathrm{X}$ \\
\hline
\end{tabular}

Note: **Correlation is significant at the 0.01 level (two-tailed) using Pearson's Product-Moment Correlation

Table 10.

Correlations between the six factors

Factors

Missing values (\%)

Person-centered care within a hospital unit Total

Person-centered care between hospital units

Continuous learning between hospital units

Prerequisites for successful patient transfers between hospital units

1.2

3.6

10.7

7.1

Table 11.

Prerequistes for successul patient transfers between hospital units

\begin{tabular}{|c|c|c|c|c|}
\hline \multirow[b]{2}{*}{ Factors } & \multicolumn{3}{|c|}{ Missing values (\%) } & \multirow{6}{*}{$\begin{array}{r}\text { Table } 12 \\
\text { Factors with missing } \\
\text { values divided by } \\
\text { profession }\end{array}$} \\
\hline & Physicians & Nurses & Assistant nurses & \\
\hline Person-centered care within a hospital unit & 5.3 & 0.0 & 0.0 & \\
\hline Person-centered care between hospital units & 0.0 & 0.0 & 8.6 & \\
\hline Continuous learning between hospital units & 0.0 & 6.7 & 20.0 & \\
\hline \multirow[t]{2}{*}{ Prerequisites for successful patient transfers between hospital units } & 0.0 & 6.7 & 11.4 & \\
\hline & & Missing & values $(\%)$ & \\
\hline Factors & ICU hos & pital 1 & ICU hospital 2 & \\
\hline Person-centered care within a hospital unit & 0. & 0 & 2.2 & Table 13. \\
\hline Person-centered care between hospital units & 7. & 7 & 0.0 & Factors with missing \\
\hline Continuous learning between hospital units & 12. & & 8.9 & values divided by \\
\hline Prerequisites for successful patient transfers between hospital units & 10. & & 4.4 & ICU and hospital \\
\hline
\end{tabular}


IJQSS

13,1

Further, this study also aimed to analyze the results to see how the survey could help improve team collaboration within ICU transitional care. The outcomes from the survey showed statistically significant differences between the two main areas for team collaboration. The main area "Team collaboration within unit" had higher mean values than the main area "Team collaboration between units." This indicates that there seems to be a fragmented picture among the coworkers of how "Person-centered care," "Continuous learning" and "Prerequisites for successful patient transfers", is perceived within and between units in the studied environment.

"Person-centered care" means that the individual needs of the patient should be taken care of by the coworkers together, both within and across organizational boundaries. This can be compared to QM and theory of team collaboration where common goals and the focus of the customer is important (Hackman and Wageman, 2005; Bronstein, 2003). Singer et al. (2015) states that a good learning environment plays an important role in improving healthcare quality and safety and that ICU transitional care appears to be influenced by teams collaborating across hospital boundaries. The results from the survey showed that coworkers perceived "Person-centered care within a unit" as stronger and more solid than they perceived "Continuous learning between units." In the same way was "Person-centered care within unit" perceived as stronger than "Person-centered care between units." The same was identified for "Continuous learning within unit" and "Continuous learning between units." Thus, there seems to be a lack of perceived "Person-centered care" and "Continuous learning," mainly between units.

The results presented showed a statistically significant difference between nurses and assistant nurses according to perceived "Person-centered care between units" and "Prerequisites for successful patient transfers between units." Different views between departments and professions in how person-centered care, coordination, communication, structure and fact-based decisions exist can lead to coworkers striving in the opposite directions with a risk for decreased patient safety. In a review by Manser (2009), the author summarizes safety-relevant aspects of teamwork, i.e. quality of collaboration, shared mental models, coordination, communication and leadership. This is in line with research by Reader et al. (2009), which conclude that communication, coordination, decision-making and leadership are processes that are shown to be important for team outputs. Also, Weller et al. (2014) conclude that challenges to increase the quality of effective teamwork in health-care organizations can include ways in which different disciplines are trained, psychological factors, the culture and the administration. The results from this study can give important insights in how team collaboration can be improved. Having a patient focus, a system view and a process perspective when setting common goals for care teams and when coordinating, communicating, structuring and deciding in ICU transitional care, may lead to a higher perceived and performed person-centered care and a more fluent care process across departments and professions. This may result in more satisfied coworkers and to increased and quality of care and patient safety.

When analyzing missing values, the factor "Continuous learning between units" had the highest degree of missing values $(10.7 \%)$, next to "Prerequisites for successful patient transfers between units" (7.1\%). Those results may indicate that there is a lack of a learning environment and prerequisites between hospital units and that the respondents do not perceive that this exist and therefore are not answering. The profession that accounted for the highest degree of missing values was assistant nurses, and this outcome was particularly high within the factor for "Continuous learning between units" (20\%). The assistant nurses at the two ICUs may call for a higher degree of learning between units. Reflecting on performed team collaboration to increase care quality and patient safety, and 
sharing experiences and knowledge together and over organizational boundaries should be a priority. This is in line with the study by Lyubovnikova et al. (2014) who state that working in "real teams" requires shared goals, interdependence, flexibility and reflexivity.

Collaboration between two research disciplines, namely, Nursing Science and QM, can give new insights, both when developing a questionnaire and when studying how team collaboration is perceived and can be improved in ICU transitional care.

\section{Methodological considerations}

To validate the new questionnaire including the main areas and factors about team collaboration, the questionnaire was first read through by a university staff member within Nursing Science, who has also practically worked as a nurse. Feedback from the test included wording, the order of the questions and clarification of sentences. Minor changes were made to clarify sentences and words.

Before handing out the questionnaire, the researchers held short information sessions about the questionnaire at staff meetings. The information included that participation was optional, that the results would be handled with confidentiality and instructions on how to fill in the questionnaire. During the information session and when completing the questionnaire, the respondents had the opportunity to ask questions about the questionnaire. The staff who did not participate at the meeting and received the questionnaire from their leaders did not have the same opportunity to ask questions directly to the researchers. This is something that might have affected how coworkers answered and if they preferred to choose the option "Don't know." Thus, they had the possibility to contact the researchers via e-mail or phone or to ask their leaders, if some questions were not clear for them.

Missing values were analyzed and were dominant for the main area "Team collaboration between units." There can be numerous causes for missing values. The respondents might not understand the statements or they might not recognize it as applicable in their context. The latter could be the case when the respondents do not perceive that there is continuous learning between units or a person-centered view of care and therefore do not answer or know what to answer. The causes of missing values in this study need further analysis.

\section{Conclusions and future research}

Measuring team collaboration in a complex context such as ICU transitional care, where multiple professions are collaborating can give insights in areas that need to be improved, to create continuity of care and to increase care quality and patient safety. From this study, several conclusions can be drawn as follows:

(1) Team collaboration in ICU transitional care can be measured with help of the developed and tested questionnaire.

(2) Measuring team collaboration can provide an understanding of how collaboration is perceived, what weaknesses there are and how it can be improved. The results from the survey gave insights that can be useful when improving team collaboration in ICU transitional care.

(3) The results indicated that teams collaborating over hospital units need to focus more on how to increase person-centered care and continuous learning and how to create prerequisites for successful patient transfers between hospital units. The results also showed that there seems to be a need for a more holistic view of team collaboration across organizational boundaries. 
IJQSS

13,1

(4) Team collaboration in this care process requires deeper understanding and mutual learning between professions. According to the results, there seems to be a lack of sharing, understanding and using knowledge during the whole process. Increased learning between professions requires an increased degree of system thinking and less thinking in silos by using knowledge from coworkers across organizational boundaries.

(5) Collaborating in teams over hospital units along the ICU transitional care process requires a common view of person-centered care, shared objectives, reflection over team performance and knowledge in how the coworkers in teams are interdependent on each other to succeed.

(6) The collaboration between two research subjects, Nursing Science and QM, has given new perspectives on how to develop a measurement tool for measuring team collaboration within ICU transitional care. Measuring perceived team collaboration can give a deeper understanding of how cultural and systemic differences and opportunities can help improving team collaboration in the ICU transitional care process by shifting focus from the individual to team, culture, system, process and continuous improvement.

\section{Future research}

The developed measurement tool was tested at two ICUs, which represent one part of the ICU transitional care process. The next step will be testing the instrument at general wards. Adding those results together will give a holistic view of perceived team collaboration and which factors that might need to be improved within the ICU transitional care process.

Another proposal for future research can be adding results from performed focus group discussions with coworkers at the ICUs and general wards. These empirical data might give new perspectives and contribute to changes in or adding statements or factors to a later version of this measurement tool.

\section{References}

Bäckström, I. and Ingelsson, P. (2016), "Measuring appreciative inquiry, lean and perceived co-worker health", Quality Innovation Prosperity, Vol. 20 No. 2, pp. 105-118.

Bergman, B. and Klefsjö, B. (2010), Quality: From Customer Needs to Customer Satisfaction, 3rd ed., Studentlitteratur, Lund.

Boughzala, I. and De Vreede, G.-J. (2015), "Evaluating team collaboration quality: the development and field application of a collaboration maturity model", Journal of Management Information Systems, Vol. 32 No. 3, pp. 129-157.

Bronstein, L.R. (2002), "Index of interdisciplinary collaboration”, Social Work Research, Vol. 26 No. 2, pp. 113-123.

Bronstein, L.R. (2003), “A model for interdisciplinary collaboration”, Social Work, Vol. 48 No. 3, pp. 297-306.

Chaboyer, W., James, H. and Kendall, M. (2005), "Transitional care after the intensive care unit: current trends and future directions", Critical Care Nurse, Vol. 25 No. 3, pp. 16-26.

Chatman, J.A. and Eunyoung Cha, S. (2003), "Leading by leveraging culture", California Management Review, Vol. 45 No. 4, pp. 20-34.

Crilly, J., Chaboyer, W. and Wallis, M. (2006), "Continuity of care for acutely unwell older adults from nursing homes”, Scandinavian Journal of Caring Sciences, Vol. 20 No. 2, pp. 122-134. 
Dahlgaard, J.J. and Dahlgaard-Park, S.M. (1999), "Integrating business excellence and innovation management: developing a culture for innovation, creativity and learning", Total Quality Management, Vol. 10 Nos 4/5, pp. 465-472.

Dahlgaard, J.J., Pettersen, J. and Dahlgaard-Park, S.M. (2011), “Quality and lean health care: a system for assessing and improving the health of healthcare organisations", Total Quality Management and Business Excellence, Vol. 22 No. 6, pp. 673-689.

Drotz, E. and Pokinska, B. (2014), "Lean in healthcare from employees" perspectives", Journal of Health Organization and Management, Vol. 28 No. 2, pp. 177-195.

Hackman, J.R. and Wageman, R. (2005), “A theory of team coaching”, Academy of Management Review, Vol. 30 No. 2, pp. 269-287.

Häggström, M. and Bäckström, B. (2014), "Organizing safe transitions from intensive care”, Nursing Research and Practice, Vol. 2014, pp. 1-11.

Häggström, M., Asplund, K. and Kristiansen, L. (2009), "Struggle with a gap between intensive care units and general wards", International Journal of Qualitative Studies on Health and Well-Being, Vol. 4 No. 3, pp. 181-192.

Häggström, M., Asplund, K. and Kristiansen, L. (2012), "How can nurses facilitate patient's transitions from intensive care?", Intensive and Critical Care Nursing, Vol. 28 No. 4, pp. 224-233.

Häggström, M., Asplund, K. and Kristiansen, L. (2013), "To reduce technology prior discharge from intensive care - important but difficult? A grounded theory", Scandinavian Journal of Caring Sciences, Vol. 27 No. 3, pp. 506-515.

Hair, J.F. (2019), Multivariate Data Analysis, 8th ed., Cengage, Andover.

Henri, J.F. (2006), "Organizational culture and performance measurement systems", Accounting, Organizations and Society, Vol. 31 No. 1, pp. 77-103.

Hollenbeck, J.R., Beersma, B. and Schouten, M.E. (2012), "Beyond team types and taxonomies: a dimensional scaling conceptualization for team description", The Academy of Management Review, Vol. 37 No. 1, pp. 82-106.

Kaissi, A., Johnson, T. and Kirschbaum, M.S. (2003), "Measuring teamwork and patient safety attitudes of high-risk areas", Nursing Economics, Vol. 21 No. 5, pp. 211-218.

Kauppi, W., Proos, M. and Olausson, S. (2018), "Ward nurses' experiences of the discharge process between intensive care unit and general ward", Nursing in Critical Care, Vol. 23 No. 3, pp. 127-133.

Kydona, C.K., Malamis, G., Giasnetsova, T., Tsiora, V. and Gritsi-Gerogianni, N. (2010), "The level of teamwork as an index of quality in ICU performance”, Hippokratia, Vol. 14 No. 2, pp. 94-97.

Lin, F., Chaboyer, W., Wallis, M. and Miller, A. (2013), "Factors contributing to the process of intensive care patient discharge: an ethnographic study informed by activity theory", International Journal of Nursing Studies, Vol. 50 No. 8, pp. 1054-1066.

Lyubovnikova, J., West, M.A., Dawson, J.F. and Carter, M.R. (2014), "24-Karat or fool's gold? Consequences of real team and co-acting group membership in healthcare organizations", European Journal of Work and Organizational Psychology, Vol. 24 No. 6, pp. 929-950.

Manser, T. (2009), "Teamwork and patient safety in dynamic domains of healthcare: a review of the literature”, Acta Anaesthesiologica Scandinavica, Vol. 53 No. 2, pp. 143-151.

Meleis, A.I. (2018), Theoretical Nursing - Development and Progress, 6th ed., Wolters Kluwers, Philadelphia.

O'leary, M.B., Mortensen, M. and Woolley, A. (2011), "Multiple team membership: a theoretical model of its effects on productivity and learning for individuals and teams", The Academy of Management Review, Vol. 36 No. 3, pp. 461-478. 
IJQSS

13,1

Oliver, D.P., Wittenberg-Lyles, E.M. and Day, M. (2007), "Measuring interdisciplinary perceptions of collaboration on hospice teams", American Journal of Hospice and Palliative Medicine $\mathbb{R} \mathbb{R}$, Vol. 24 No. 1, pp. 49-53.

Reader, W.T., Flin, H.R., Mearns, H.K. and Cutherbertson, H.B. (2009), "Developing a team performance framework for the intensive care unit", Critical Care Medicine, Vol. 37 No. 5, pp. 1787-1793.

Renton, J., Pilcher, D., Santamaria, J., Stow, P., Bailey, M., Hart, G. and Duke, G. (2011), "Factors associated with increased risk of readmission to intensive care in Australia", Intensive Care Medicine, Vol. 37 No. 11, pp. 1800-1808.

Schein, E.H. (2009), The Corporate Culture Survival Guide, Jossey-Bass, San Francisco, CA.

Sharp, S., Mcallister, M. and Broadbent, M. (2018), "The tension between person centred and task focused care in an acute surgical setting: a critical ethnography", Collegian, Vol. 25 No. 1, pp. 11-17.

Singer, S.J., Benzer, J.K. and Hamdan, S.U. (2015), "Improving health care quality and safety: the role of collective learning", Journal of Healthcare Leadership, Vol. 7, pp. 91-107.

Stelfox, H.T., Lane, D., Boyd, J.M., Taylor, S., Perrier, L., Straus, S., Zygun, D. and Zuege, D.J. (2015), “A scoping review of patient discharge from intensive care opportunities and tools to improve care", Chest, Vol. 147 No. 2, pp. 317-327.

Sundstrom, E., De Meuse, K.P. and Futrell, D. (1990), "Work teams: applications and effectiveness", American Psychologist, Vol. 45 No. 2, pp. 120-133.

Tavakol, M. and Dennick, R. (2011), "Making sense of Cronbach's alpha”, International Journal of Medical Education, Vol. 2, pp. 53-55.

Van Sluisveld, N., Oerlemans, A., Westert, G., Van der Hoeven, J.G., Wollersheim, H. and Zegers, M. (2017), "Barriers and facilitators to improve safety and efficiency of the ICU discharge process: a mixed methods study", BMC Health Service Research, Vol. 17 No. 1, pp. 1-12.

Vyt, A. (2008), "Interprofessional and transdisciplinary teamwork in health care", Diabetes/Metabolism Research and Reviews, Vol. 24 No. S1, pp. S106-S109.

Weller, J., Boyd, M. and Cumin, D. (2014), "Teams, tribes and patient safety: overcoming barriers to effective teamwork in healthcare", Postgraduate Medical Journal, Vol. 90 No. 1061, pp. 149-154.

West, M.A. and Lyubovnikova, J. (2013), "Illusions of team working in health care", Journal of Health Organization and Management, Vol. 27 No. 1, pp. 134-142.

West, M.A. and Lyubovnikova, J. (2012), "Real teams or pseudo teams? The changing landscape needs a better map", Industrial and Organizational Psychology, Vol. 5 No. 1, pp. 25-28.

Whittaker, J. and Ball, C. (2000), "Discharge from intensive care: a view from the ward", Intensive and Critical Care Nursing, Vol. 16 No. 3, pp. 135-143.

\section{Further reading}

World Health Organization (WHO) (2020), "Patient safety 2030", available at: www.who.int/ patientsafety/news_events/ps-2030-report.pdf (accessed 10 March 2020).

\section{Corresponding author}

Lilly-Mari Sten can be contacted at: lilly-mari.sten@miun.se

For instructions on how to order reprints of this article, please visit our website:

www.emeraldgrouppublishing.com/licensing/reprints.htm

Or contact us for further details: permissions@emeraldinsight.com 